\title{
Asynchronous LOS Discovery Algorithm for Aerial Nodes Using In-band Full-Duplex Transceivers
}

\author{
A F M Saniul Haq \\ University of Central Florida \\ saniul.haq@knights.ucf.edu
}

\author{
Mahmudur Khan \\ The University of Alabama \\ mkhan6@ua.edu
}

\author{
Murat Yuksel \\ University of Central Florida \\ murat.yuksel@ucf.edu
}

\begin{abstract}
Emerging communication techniques in terahertz bands, including free-space optical (FSO), have the potential to aid in solving the spectrum scarcity problem by complementing the traditional radio frequency (RF) wireless networks. Line-of-Sight (LOS) link discovery is one of the major limiting factors for these highly directional bands, and this problem of establishing LOS between neighbor nodes becomes more challenging when they have no apriory knowledge of the location of each other. In this work, we present an asynchronous LOS discovery algorithm using spiral scanning without any synchronization via an RF channel. We also demonstrate the effectiveness of the algorithm through test-bed experiments using a prototype of UAVs equipped with in-band full-duplex (IBFD) FSO transceivers.
\end{abstract}

\section{CCS CONCEPTS}

- Hardware $\rightarrow$ Wireless integrated network sensors; Wireless devices; $\bullet$ Computer systems organization $\rightarrow$ Sensor networks;

\section{KEYWORDS}

In-band full duplex transceiver, optical wireless communication, asynchronous LOS discovery, free space optics

\section{INTRODUCTION}

As part of the terahertz bands, free-space optical (FSO), a.k.a. optical wireless, communication is a promising and emerging complementary alternative to the legacy radio frequency (RF) bands to satiate the ever increasing need for wireless data. FSO transceivers offer high data transmission rates, directionality, longer lifetime, and cheap integration for mass deployment $[4,6]$. The huge unlicensed optical spectrum and significantly lower transmit power per bit property make FSO communication (FSOC) a great opportunity for future spectrum-scarce mobile networks and power-hungry Internet-of-Things (IoT) systems [7]. Low-intercept characteristics of FSOC due to its high directionality and dense integration in lightweight systems are also attractive for secure wireless communications using UAVs or drones [2].

FSO-enabled UAVs are envisioned to play a significant role in future generation mobile wireless ad hoc networks. Swarms of UAVs connected to each other via FSOC links can help to relay data

Permission to make digital or hard copies of part or all of this work for personal or classroom use is granted without fee provided that copies are not made or distributed for profit or commercial advantage and that copies bear this notice and the full citation on the first page. Copyrights for third-party components of this work must be honored.

For all other uses, contact the owner/author(s).

CoNEXT'19 Companion, December 9-12,2019, Orlando, FL, USA

(c) 2019 Copyright held by the owner/author(s).

ACM ISBN 978-1-4503-7006-6/19/12 . \$15.00

https://doi.org/10.1145/3360468.3366777 from pico-cells to the core network. UAVs are also used for both civil and military missions, such as monitoring of an area hit by a natural disaster, broadcasting data at some critical sports event or even observing behind the enemy lines. Recently, Alphabet Inc. deployed stratospheric solar-powered balloons to provide Internet service to remote areas of Puerto Rico where cellphone towers were damaged by Hurricane Maria. All these different applications of UAVs produce large amounts of data that is required to be delivered to a ground station or other UAVs [8]. Using FSO transceivers, these large volumes of data can be transferred at extremely high speeds.

In this work, we tackle the problem of LOS link discovery using directional FSOC links between nodes in 3D space. We assume that the nodes can mechanically steer their respective transceivers with randomly chosen angular speeds from a randomly selected position on a spiral path and perform a three-way handshake to discover each other on an LOS link. The nodes change their angular speeds if the discovery is not successful within an optimal time interval, which is set by performing a number of test runs by placing each node at random positions. We evaluate the performance of the proposed approach through real test-bed experiments. The results show that, using such mechanically steerable highly directional transceivers, two neighbor nodes can discover each other successfully even without prior location information of each other. Key insights and contributions of this work include:

- An in-band method for two nodes in 3D to discover each other without prior knowledge of the neighbor's location using only highly directional transceiver.

- A heuristic protocol that chooses an angular speed randomly, and updates the speed after threshold times.

- We observed from the experiments that neighbor discovery is possible using in-band full-duplex directional transceivers.

\section{SYSTEM DESIGN AND EVALUATION}

We explored asynchronous spiral neighbor discovery algorithm to establish a LOS link between two aerial vehicles. The nodes are positioned randomly in 3D space and each node carries an IBFD FSO transceiver mounted on servo motors. The transceiver is designed using two commercially available IrDA2 units to ensure full-duplex configuration $[1,5]$. No additional omni-directional RF channel is used for synchronization or data transmission. The neighbor discovery method is to scan the surrounding 3D space by rotating the transceivers in randomly chosen spiral paths in 3D [5, 9]. Each node arbitrarily chooses an angular speed $(\omega)$ and a start position $(P(\phi, \theta))$ on the predefined spiral path to start the discovery process. LOS discovery is completed when they are able to perform a 3-way handshake between the two nodes. Figure 1(a) shows the path of the transceiver head for spiral scanning. For asynchronous discovery, 


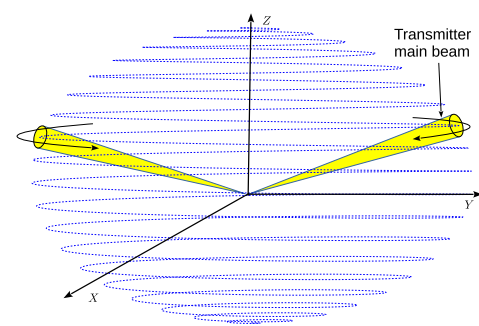

(a)

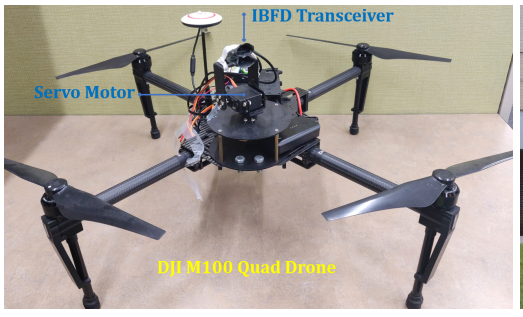

(b)

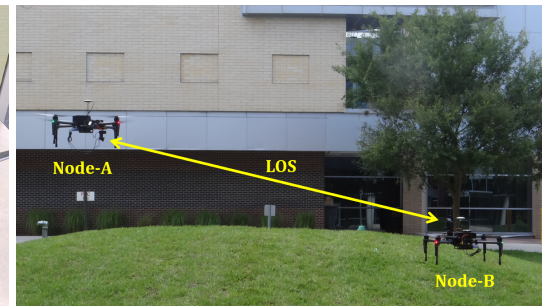

(c)

Figure 1: (a) Spiral search space for the transceiver, (b)-(c) System setup for asynchronous spiral LOS discovery experiments.

initial start point $(P(\phi, \theta))$ is also randomly set, where $\phi$ and $\theta$ are angles w.r.t. $z$-axis and $x$-axis, respectively.

One of the corner cases of asynchronous LOS discovery is the case when angular speeds $(\omega)$ of the two nodes are same or very similar, which may result in longer discovery time [3]. By choosing random initial start point, we can increase the probability of discovery. But it is also important to reassign a new value to $\omega$ after a certain reset time $\left(t_{\text {reset }}\right)$, since the benefit of randomly searching for the other node diminishes quickly. The algorithm for the LOS discovery phase using asynchronous spiral scan is given in Algorithm 1.

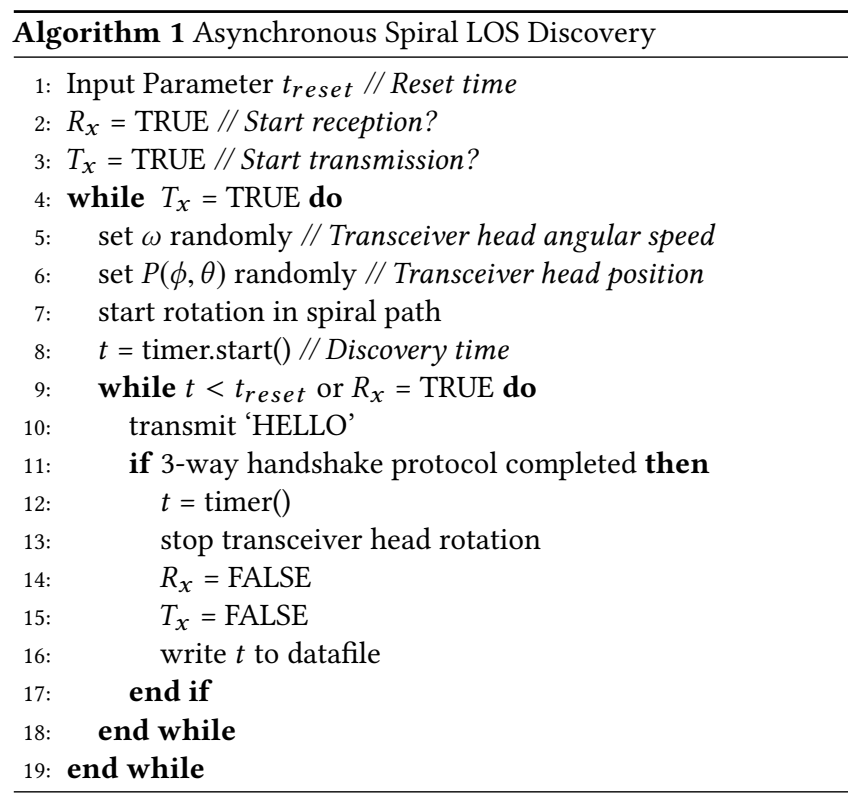

For these experiments, the angular speed, $\omega$, is chosen by a node randomly, where $\omega \in[\pi / 2, \pi] \mathrm{rad} / \mathrm{s}$. The range of available $\omega$ is limited by the capabilities of the servo motors. For our prototype, the servo motors have maximum angular speed of $\pi \mathrm{rad} / \mathrm{s}$. To determine the reset time, $t_{\text {reset }}$, we conducted multiple LOS discovery runs and chose $t_{\text {reset }}$ for a given confidence level $(\alpha)$ from the empirical distribution.

Figure 2 shows the distribution and CDF of the LOS discovery time, repeated for 111 times, while the drone was hovering. From the experimental data, we can see that average discovery time is

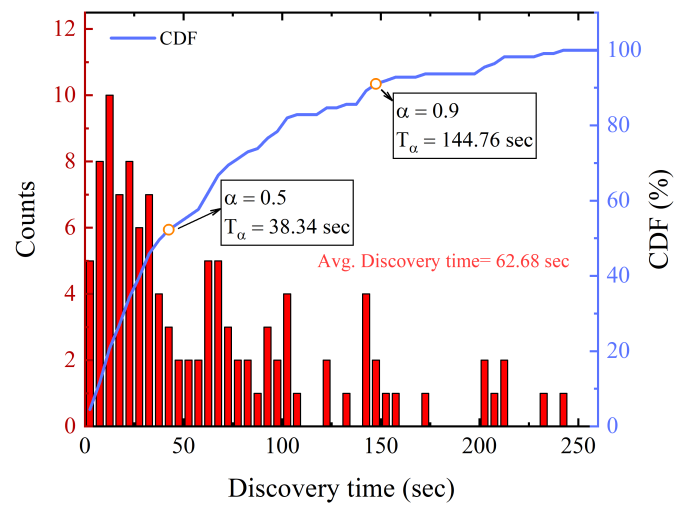

Figure 2: Empirical discovery time distribution.

$62.68 \mathrm{sec}$ and discovery is completed within $144.76 \mathrm{sec} 90 \%$ of the time, whereas it is completed within $38.34 \mathrm{sec} 50 \%$ of the time. It should be noted here, the reset time can be different for different confidence level $(\alpha)$ and divergence angle $(\beta)$. We have listed the calculated reset times for different $\alpha$ from the empirical distribution in Table 1. The average discovery time for a two-node system can be further improved by optimizing $t_{\text {reset }}, \omega$, and $\alpha$.

Table 1: Reset time for different confidence level

\begin{tabular}{|c|c|c|c|c|c|}
\hline$\alpha$ & 0.1 & 0.3 & 0.5 & 0.7 & 0.9 \\
\hline \hline$t_{\text {reset }}(\mathrm{sec})$ & 6.31 & 19.56 & 38.39 & 74.26 & 144.76 \\
\hline
\end{tabular}

\section{CONCLUSION}

In summary, we demonstrated that two UAVs can discover each other by mechanically steering their IBFD directional transceivers using an asynchronous discovery protocol by performing real testbed experiments. We demonstrated that by randomly selecting angular speed and initial scan position, the LOS link can be established between two UAVs within a limited time period. The experiments for improving the discovery time by resetting angular scan speed after a threshold time $\left(t_{\text {reset }}\right)$ is still in progress. Another promising line of future work is to extend the capabilities of the algorithm beyond discovery phase into the maintenance phase, where the established LOS can be maintained even the aerial nodes are in motion and within communication range.

\section{ACKNOWLEDGEMENT}

This work is supported in part by U.S. National Science Foundation awards 1836741 and 1647189. 


\section{REFERENCES}

[1] AFM Saniul Haq, Mahmudur Rahman Khan, and Murat Yuksel. 2018. A Prototype of In-Band Full-Duplex Free-Space Optical Transceiver. In Proceedings of IEEE International Symposium on Local and Metropolitan Area Networks (LANMAN). IEEE, 112-113.

[2] Mahmudur Khan and Murat Yuksel. 2015. Autonomous Alignment of Free-SpaceOptical Links Between UAVs. In Proceedings of the 2nd International Workshop on Hot Topics in Wireless. ACM, 36-40.

[3] Mahmudur Khan and Murat Yuksel. 2018. In-band LOS discovery between drones using highly directional transceivers. In Proceedings of the 4th ACM Workshop on Micro Aerial Vehicle Networks, Systems, and Applications. ACM, 51-56.

[4] Mahmudur Khan, Murat Yuksel, and Garrett Winkelmaier. 2017. GPS-free maintenance of a free-space-optical link between two autonomous mobiles. IEEE Transactions on Mobile Computing 16, 6 (2017), 1644-1657.

[5] Mahmudur Rahman Khan, Suman Bhunia, Murat Yuksel, and Lawrence Kane 2018. Line-of-Sight Discovery in 3D Using Highly Directional Transceivers. IEEE Transactions on Mobile Computing (2018).

[6] Chin Wan Joanne Oh, Zizheng Cao, Eduward Tangdiongga, and Ton Koonen 2016. 10 Gbps all-optical full-duplex indoor optical wireless communication with wavelength reuse. In Proceedings of Optical Fiber Conference. OSA, Th4A-6.

[7] Ke Wang, Ampalavanapillai Nirmalathas, Christina Lim, Kamal Alameh, and Efstratios Skafidas. 2016. Full-duplex Gigabit indoor optical wireless communication system with CAP modulation. IEEE Photonics Technology Letters 28, 7 (2016), 790-793.

[8] Dyke Weatherington and U Deputy. 2005. Unmanned aircraft systems roadmap, 2005-2030. UAV Planning Task Force, OUSD (AT\&L) (2005)

[9] Zhensheng Zhang and Bo Li. 2008. Neighbor discovery in mobile ad hoc selfconfiguring networks with directional antennas: Algorithms and comparisons. IEEE Transactions on Wireless Communications 7, 5 (2008) 